\title{
Effect of boundaries on the spectrum of a one-dimensional random mass Dirac Hamiltonian
}

\author{
Christophe Texier ${ }^{(a, b)}$ and Christian Hagendorf ${ }^{(c)}$
}

November 6, 2009

(a) Laboratoire de Physique Théorique et Modèles Statistiques, UMR 8626 du CNRS, Université Paris-Sud, Bât. 100, F-91405 Orsay Cedex, France.

(b) Laboratoire de Physique des Solides, UMR 8502 du CNRS, Université Paris-Sud, Bât. 510, F-91405 Orsay Cedex, France.

(c) Laboratoire de Physique Théorique de l'École Normale Supérieure, 24, rue Lhomond, F-75230 Paris Cedex 05, France.

\begin{abstract}
The average density of states (DoS) of the one-dimensional Dirac Hamiltonian with a random mass on a finite interval $[0, L]$ is derived. Our method relies on the eigenvalues distributions (extreme value statistics problem) which are obtained explicitly. The well-known Dyson singularity $\overline{\varrho(\epsilon ; L)} \sim-\frac{L}{|\epsilon| \ln ^{3}|\epsilon|}$ is recovered above the crossover energy $\epsilon_{c} \sim \exp -\sqrt{L}$. Below $\epsilon_{c}$ we find a log-normal suppression of the average DoS $\overline{\varrho(\epsilon ; L)} \sim \frac{1}{|\epsilon| \sqrt{L}} \exp \left(-\frac{1}{L} \ln ^{2}|\epsilon|\right)$.
\end{abstract}

PACS numbers : 72.15.Rn ; 73.20.Fz ; 02.50.-r.

\section{Introduction}

Let us consider the one-dimensional Dirac equation $\mathcal{H}_{\mathrm{D}} \psi=\epsilon \psi$ for the Hamiltonian

$$
\mathcal{H}_{\mathrm{D}}=-\alpha_{x} \mathrm{i} \partial_{x}+\beta \phi(x)
$$

acting on a two-component spinor. $\phi(x)$ plays the role of a mass and will be taken as random. The Dirac matrices are chosen as $\beta=\gamma^{0}=\sigma_{1}$ and $\alpha_{x}=\gamma^{0} \gamma^{1}=-\sigma_{2}$, where $\sigma_{i}$ are the usual Pauli matrices, corresponding to the representation of the Clifford algebra $\gamma^{0}=\sigma_{1}$ and $\gamma^{1}=-\mathrm{i} \sigma_{3}$. One-dimensional random Dirac Hamiltonians appear in several contexts of condensed matter physics ranging from disordered half-filled metals [1, 2], random spin-chain models (random antiferromagnetic spin-1/2 chains [3, 4, 5], random transverse field Ising spin- $1 / 2$ chains [6, 7, 8, spin-Peierls chains and spin-ladders [9, 10]), or organic conductors [11]. Another common representation of Dirac matrices $\beta=\sigma_{1}$ and $\alpha_{x}=\sigma_{3}$, related to the one chosen in the present article by a unitary transformation $\mathcal{U}=\left(1+\mathrm{i} \sigma_{1}\right) / \sqrt{2}$, shows that the Dirac equation has the form of the linearised Bogoliubov-de Gennes equation describing a superconductor with random gap [12, 13. We obtain a connection to another important, and well-studied problem by squaring the Dirac Hamiltonian: $\mathcal{H}_{\mathrm{D}}^{2}=-\partial_{x}^{2}+\phi(x)^{2}+\mathrm{i} \gamma^{1} \phi^{\prime}(x)=-\partial_{x}^{2}+\phi(x)^{2}+\sigma_{3} \phi^{\prime}(x)$. This leads to a couple of isospectral supersymmetric Hamiltonians $H_{ \pm}=-\frac{\mathrm{d}^{2}}{\mathrm{~d} x^{2}}+\phi(x)^{2} \pm \phi^{\prime}(x)$. The corresponding Schrödinger equation can be transformed into a Fokker-Planck equation $\partial_{t} P=\partial_{x}\left(\partial_{x} \mp 2 \phi(x)\right) P$ describing classical diffusion in random force field, studied in numerous 
works [14, 15, 16, 17, 18, 8, 19, 20. If the random mass is a Gaussian white noise with characteristics $\overline{\phi(x)}=\mu g$ and $\overline{\phi(x) \phi\left(x^{\prime}\right)}-\bar{\phi}^{2}=g \delta\left(x-x^{\prime}\right)$, where $\cdots$ denotes the average with respect to its realisations, an exact analytical expression for the density of states (DoS) was obtained [12, 17] (see $\S 8.2$ of Ref. [13]) 1]. Another solvable case is the one where the mass is chosen as a telegraph noise [22] (i.e. with exponentially decaying correlations). The same low energy properties are obtained. This is related to the fact that any short-range correlated noise, upon large scale renormalization, reduces to a Gaussian white noise.

Here we focus on the case of Gaussian white noise with $\overline{\phi(x)}=0$ which is known to yield a Dyson singularity for the $\operatorname{DoS} \varrho(\epsilon \rightarrow 0) \simeq \frac{g}{|\epsilon| \ln ^{3}(g /|\epsilon|)}$ and a delocalisation transition at $\epsilon \rightarrow 0$. The aim of the present article is to study how the Dyson singularity of the DoS is affected by boundary conditions. We denote by $\varrho(\epsilon ; L)$ the DoS of the Hamiltonian (1) on a finite interval $[0, L]$. We will obtain the behaviour of the average DoS $\overline{\varrho(\epsilon ; L)}$ for a finite length.

\section{Boundary conditions}

Let us specify the two different types of boundary conditions we shall consider.

Type $(D)$ : A simple way to introduce boundary conditions ensuring confinement in a domain $\mathcal{D}$ is to consider the Dirac equation $[\mathrm{i} \not \partial-\phi(x)] \psi(x, t)=0$ with infinite mass $\phi(x)=\Phi \rightarrow \infty$ for $x$ outside $\mathcal{D}$. This leads to the so-called "bag model" of hadronic physics [23] : the boundary conditions are $\left.(1+\mathrm{i} \vec{n} \cdot \vec{\gamma}) \psi\right|_{\partial \mathcal{D}}=0$, where $\vec{n}$ is the unit vector normal at the boundary $\partial \mathcal{D}$ of the domain. They force the vanishing of the component of the current density $\overrightarrow{\mathcal{J}}_{\psi}=\bar{\psi} \vec{\gamma} \psi$ perpendicular to the boundary, where $\bar{\psi}=\psi^{\dagger} \gamma^{0}$. In our case, for $x \in \mathbb{R}^{-}$the stationary solution of the Dirac equation for a constant mass is a plane wave $\psi(x)=u \mathrm{e}^{\mathrm{i} k x}$ where the spinor $u$ is solution of $\left(\alpha_{x} k+\beta \Phi\right) u=\epsilon u$, for $\epsilon^{2}=k^{2}+\Phi^{2}$. In the limit $\Phi \rightarrow \infty$ it becomes an evanescent wave with $k \simeq-\mathrm{i} \Phi$; this yields $\left(1-\mathrm{i} \gamma^{1}\right) \psi(0)=0$. Similarly, at the other boundary we find $\left(1+\mathrm{i} \gamma^{1}\right) \psi(L)=0$. We denote these constraints as "type (D) boundary conditions". With our representation of the Clifford algebra, they read $\left(1-\sigma_{3}\right) \psi(0)=\left(1+\sigma_{3}\right) \psi(L)=0$, i.e. each of the two components of the bispinor $\psi=(\varphi, \chi)$ must vanish at one side : $\chi(0)=\varphi(L)=0$. This condition obviously ensures the absence of Dirac current flow $\mathcal{J}_{\psi}=-\psi^{\dagger} \sigma_{2} \psi$ across the boundaries.

Type $(S)$ : A second interesting choice is given by $\left(1+\sigma_{3}\right) \psi(0)=\left(1+\sigma_{3}\right) \psi(L)=0$. These conditions coincide with Dirichlet boundary conditions for the associated supersymmetric Schrödinger Hamiltonian $H_{ \pm}$and will be denoted as "type (S)" 2 .

\footnotetext{
${ }^{1}$ Note also the Ref. [21] where the most general 1d Dirac Hamiltonian $\mathcal{H}_{\mathrm{D}}=\sigma_{2} \mathrm{i} \frac{\mathrm{d}}{\mathrm{d} x}+\sigma_{1} \phi(x)+\sigma_{3} \lambda(x)+V(x)$ was studied for $\phi, \lambda$ and $V$ Gaussian white noises. This study showed that there is no other DoS singularity than the one arising in the random mass case.

${ }^{2}$ General boundary conditions : It is possible to set up more general conditions forcing the absence of probability current flow at the boundaries : these are the set of conditions parametrised by some real number $\vartheta$ [24] : $\left.\left(\mathrm{e}^{\mathrm{i} \vartheta \gamma^{5}}+\mathrm{i} \vec{n} \cdot \vec{\gamma}\right) \psi\right|_{\partial \mathcal{D}}=0$ where $\gamma^{5}$ is the chirality matrix, given by $\gamma^{5}=\gamma^{0} \gamma^{1}$ in dimension $d=1+1$. These general boundary conditions are obtained as follows : one considers the Dirac equation in a bounded domain $\mathcal{D}$. The boundary conditions can be written $\left.\mathrm{i} \vec{n} \cdot \vec{\gamma} \psi\right|_{\partial \mathcal{D}}=\left.\left(A+B \gamma^{5}\right) \psi\right|_{\partial \mathcal{D}}$, where $\vec{n}$ is the unit vector normal to the domain, $A$ and $B$ two complex numbers. The fact that $(\vec{n} \cdot \vec{\gamma})^{2}=-1$ leads to $A^{2}-B^{2}=1$. Upon imposing the normal component of the Dirac current to vanish $\left.\vec{n} \cdot \overrightarrow{\mathcal{J}}_{\psi}\right|_{\partial \mathcal{D}}=0$, we find $A \in \mathbb{R}$ and $B \in \mathrm{i} \mathbb{R}$, and thus the desired form. With the representation chosen in our article these general boundary conditions take the form $\left(\mathrm{e}^{-\mathrm{i} \vartheta_{ \pm} \sigma_{2}} \pm \sigma_{3}\right) \psi\left(x_{ \pm}\right)=0$ with $x_{-}=0$ and $x_{+}=L$. For type (D) we have $\vartheta_{+}=\vartheta_{-}=0$, and for $(\mathrm{S})$ $\vartheta_{-}=\pi, \vartheta_{+}=0$. Since $\vartheta_{ \pm} \neq 0$ or $\pi$ breaks the particle-hole symmetry, this case will not be considered here.
} 


\section{Eigenvalue distributions}

We now consider the Dirac Hamiltonian $(1)$ on $[0, L]$ for the two kinds of boundary conditions introduced above. The particle-hole symmetry takes the form $\psi \rightarrow \tilde{\psi}=\sigma_{3} \psi: \sigma_{3} \mathcal{H}_{\mathrm{D}} \sigma_{3}=-\mathcal{H}_{\mathrm{D}}$. Since both boundary conditions preserve the particle-hole symmetry, eigenvalues appear in pairs $\pm \epsilon_{n}$ (by convention we choose $n=1,2,3, \cdots$ and $\epsilon_{n}>0$ ). We denote by $\varrho(\epsilon)$ the DoS per unit length for an infinite volume. It is related to the DoS of the finite-size system by $\varrho(\epsilon)=$ $\lim _{L \rightarrow \infty} \varrho(\epsilon ; L) / L$. Because of particle-hole symmetry we can restrict ourselves to $\epsilon>0$.

Our method to obtain the average $\operatorname{DoS} \overline{\varrho(\epsilon ; L)}$ relies on the evaluation of the eigenvalue distributions $\mathcal{W}_{n}(\epsilon ; L) \stackrel{\text { def }}{=} \overline{\delta\left(\epsilon-\epsilon_{n}[\phi, L]\right)}$, derived in Ref. [25] for (S)-boundaries. Finding the distributions of the (ordered) variables $\epsilon_{n}$ corresponds to an "extreme value problem" (here for correlated variables). We recall the idea of the method : let us imagine that we impose the boundary conditions to hold solely at one end of the interval, say $x=0$. As $x$ increases the two components of the spinor $\psi(x)=(\varphi(x), \chi(x))$, which solves the Dirac equation, vanish alternately ; we denote by $\Lambda_{m}$ the distance between a node of $\varphi(x)$ and the closest node of $\chi(x)$. Since the evolution of $\psi(x)$ is Markovian, the $\Lambda_{m}$ 's are identical and independently distributed (i.i.d.) random variables whose statistical properties are obtained by solving a first passage time problem (see Ref. 25] for details). Let us introduce the distribution $\pi_{M}(y)$ of the sum of $M$ i.i.d. rescaled lengths $y=\left(\Lambda_{1}+\cdots+\Lambda_{M}\right) \mathcal{N}(\epsilon)$, where

$$
\mathcal{N}(\epsilon)=\int_{0}^{\epsilon} \mathrm{d} \epsilon^{\prime} \varrho\left(\epsilon^{\prime}\right) \underset{\epsilon \rightarrow 0}{=} \frac{g / 2}{[\ln (2 g / \epsilon)-\mathrm{C}]^{2}+\pi^{2} / 4}+O\left(\frac{\epsilon^{2}}{\ln ^{2} \epsilon}\right) \approx \frac{g}{2 \ln ^{2}(g / \epsilon)}
$$

is the integrated DoS per unit length for an infinite volume ; $\mathrm{C}=0.577 \ldots$ is the Euler-Mascheroni constant (the exact expression for $\mathcal{N}(\epsilon)$ may be found in Refs. [12, 13, 17]). This rescaling is motivated by the fact that $\overline{\Lambda_{m}}=1 /(2 \mathcal{N}(\epsilon))$ [25], and hence $\bar{y}=M / 2$. Both boundary conditions at $x=0, L$ are satisfied whenever the sum $\Lambda_{1}+\cdots+\Lambda_{M}$ coincides with the length $L$. Therefore the distribution $\mathcal{W}_{n}(\epsilon ; L)$ is given by ${ }^{3}$

$$
\mathcal{W}_{n}(\epsilon ; L)=L \varrho(\epsilon) \varpi_{n}(L \mathcal{N}(\epsilon)),
$$

where the dimensionless function $\varpi_{n}(y)$ is related to the distributions $\pi_{M}(y)$ as follows :

Type $(S)$ : The same component of the Dirac spinor must vanish at the two sides of the interval. Therefore $L$ must coincide with a sum of an even number of lengths $\Lambda_{m}$ 's (see eq. (132) of Ref. [25]) :

$$
\varpi_{n}^{(S)}(y)=\pi_{2 n}(y)
$$

Type (D) : Both spinor components must vanish at one side of the interval. Hence $L$ must coincide with a sum of an odd number of lengths $\Lambda_{m}$ 's :

$$
\varpi_{n}^{(D)}(y)=\pi_{2 n-1}(y) \text {. }
$$

The distributions $\pi_{n}(y)$ are explicitly known in the low energy limit $\epsilon \ll g$, i.e. for $g L \gg 1$ where $\mathcal{W}_{n}(\epsilon ; L)$ is essentially concentrated below $g$. Indeed, the characteristic function of the lengths $\Lambda_{m}$ 's is given by [25] $\overline{\mathrm{e}^{-\alpha \Lambda}} \simeq \cosh ^{-1} \sqrt{\alpha / \mathcal{N}(\epsilon)}$. Whence we may write

$$
\pi_{n}(y) \simeq \int_{\mathcal{B}} \frac{\mathrm{d} q}{2 \mathrm{i} \pi} \frac{\mathrm{e}^{q y}}{\cosh ^{n} \sqrt{q}}
$$

\footnotetext{
${ }^{3}$ Note that the change of variable from $\epsilon$ to $L \mathcal{N}(\epsilon)$ corresponds to the so-called spectrum unfolding leading to a unit density of variables.
} 
where the integration is taken along the Bromwich contour. Starting from this integral representation we obtain the following explicit formulae

$$
\begin{gathered}
\pi_{2 k+1}(y)=\frac{2^{2 k}}{\sqrt{\pi} y^{3 / 2}} \sum_{n=0}^{\infty}(-1)^{n+k}(2 n+1)\left(\begin{array}{c}
n+k \\
n-k
\end{array}\right) \mathrm{e}^{-\frac{(n+1 / 2)^{2}}{y}} \\
\pi_{2 k}(y)=\frac{2^{2 k}}{\sqrt{\pi} y^{3 / 2}} \sum_{n=0}^{\infty}(-1)^{n+k} n\left(\begin{array}{c}
n+k-1 \\
n-k
\end{array}\right) \mathrm{e}^{-n^{2} / y}
\end{gathered}
$$

whose demonstration is provided in appendix A.

\section{DoS on a finite interval}

The average DoS can be related to the distributions recalled above :

$$
\overline{\varrho(\epsilon ; L)}=\sum_{n=1}^{\infty} \mathcal{W}_{n}(\epsilon ; L) \text {. }
$$

Summation over $n$ leads to

$$
\overline{\varrho(\epsilon ; L)}=L \varrho(\epsilon) \mathcal{D}(L \mathcal{N}(\epsilon))
$$

where the dimensionless function $\mathcal{D}(y)$ depends on boundary conditions. Note that this DoS has some interest for studying the problem of 1d classical diffusion in a random force field with dilute absorbers [26] (see also Ref. [27] for an analysis of this problem with the real space renormalisation group).

Boundary conditions of type (S).- The summation of the (S)-type distributions (4) gives

$$
\mathcal{D}_{S}(y)=\int_{\mathcal{B}} \frac{\mathrm{d} q}{2 \mathrm{i} \pi} \frac{\mathrm{e}^{q y}}{\sinh ^{2} \sqrt{q}} .
$$

The integrand is meromorphic in the complex plane (there is no branch cut since $\sqrt{q}$ is the argument of an even function). The integral can be computed from the residue theorem. The integrand possesses a single pole at $q=0$ with residue equal to unity and an infinite number of double poles on the real axis at $q=q_{n}=-(n \pi)^{2}$, with $n=1,2,3, \cdots$. Using that $\sinh ^{2} \sqrt{q} \underset{q \sim q_{n}}{\simeq}$

$\frac{1}{4 q_{n}}\left(q-q_{n}\right)^{2}$, we find the residues $\operatorname{Res}\left[\frac{\mathrm{e}^{q y}}{\sinh ^{2} \sqrt{q}} ; q_{n}\right]=\frac{\mathrm{d}}{\mathrm{d} q}\left[\frac{\left(q-q_{n}\right)^{2} \mathrm{e}^{q y}}{\sinh ^{2} \sqrt{q}}\right]_{q=q_{n}}=\left(2+4 q_{n}\right) \mathrm{e}^{q_{n} y}$. Therefore we have

$$
\mathcal{D}_{S}(y)=1+2 \sum_{n=1}^{\infty}\left(1-2(n \pi)^{2} y\right) \mathrm{e}^{-(n \pi)^{2} y}=\frac{4}{\sqrt{\pi} y^{3 / 2}} \sum_{n=1}^{\infty} n^{2} \mathrm{e}^{-n^{2} / y} .
$$

where the second series expansion may readily be found from Poisson's summation formula (see appendix B. We may also check that the summation of $7 \mathrm{~b}$ leads to $(11$.

Boundary conditions of type (D).- Compared to the (S) case, an additional cosh $\sqrt{q}$ appears in the integrand upon summation of (5) :

$$
\mathcal{D}_{D}(y)=\int_{\mathcal{B}} \frac{\mathrm{d} q}{2 \mathrm{i} \pi} \frac{\cosh \sqrt{q}}{\sinh ^{2} \sqrt{q}} \mathrm{e}^{q y}
$$




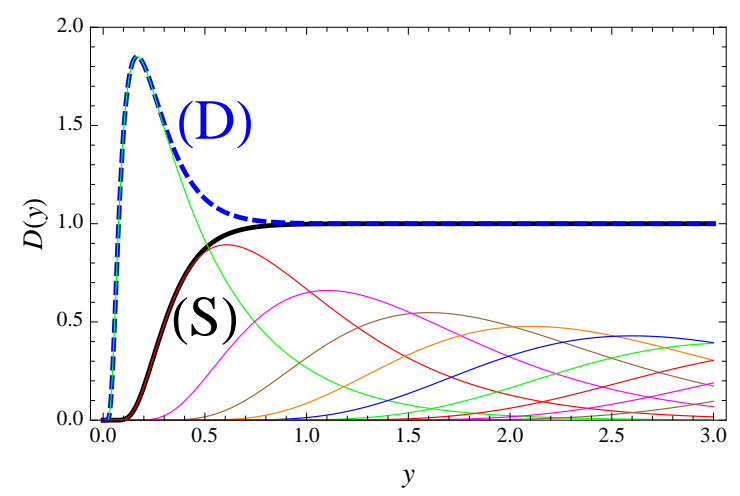

Figure 1: Functions $\mathcal{D}_{S}(y)$ (black continuous line) and $\mathcal{D}_{D}(y)$ (dashed blue line). First distributions $\varpi_{n}(y)$ are also plotted in thin lines.

It adds a sign $(-1)^{n}$ to the residues obtained in the previous case and we thus find

$$
\mathcal{D}_{D}(y)=1+2 \sum_{n=1}^{\infty}(-1)^{n}\left(1-2(n \pi)^{2} y\right) \mathrm{e}^{-(n \pi)^{2} y}=\frac{1}{\sqrt{\pi} y^{3 / 2}} \sum_{n=0}^{\infty}(2 n+1)^{2} \mathrm{e}^{-(2 n+1)^{2} / 4 y} .
$$

The two different boundary conditions can be treated on the same footing by writing

$$
\mathcal{D}_{S, D}(y)=\left(1+2 y \frac{\mathrm{d}}{\mathrm{d} y}\right) \sum_{n \in \mathbb{Z}}( \pm 1)^{n} \mathrm{e}^{-(n \pi)^{2} y}
$$

and

$$
\mathcal{D}_{S, D}(y)=\frac{4}{\sqrt{\pi} y^{3 / 2}} \sum_{n=0}^{\infty}(n+\eta)^{2} \mathrm{e}^{-\frac{(n+\eta)^{2}}{y}} \quad \text { with } \eta=\left\{\begin{array}{ll}
0 & \text { for } S \\
1 / 2 & \text { for } D
\end{array} .\right.
$$

This second series expansion allows to analyse the low energy behaviour. It is worth noticing that this representation for $\mathcal{D}(y)$ is very similar to the corresponding one for $\varpi_{1}(y)$, for both kind of boundary conditions. In the $(\mathrm{S})$ case eqs. (22) and r.h.s. of $(13)$ only differ by the $(-1)^{n+1}$ in the sum, while in the (D) case (11) and r.h.s. of $(20)$ differ by a $(-1)^{n}(2 n+1)$. As a result we have $\mathcal{D}(y \rightarrow 0) \simeq \varpi_{1}(y)$ in both cases. The two functions are plotted on figure 1 . $\mathcal{D}_{S}(y)$ presents a monotonous behaviour what leads to a diminution of the low energy DoS for small energies. Interestingly for (D) boundary conditions, while exponentially suppressed for $y \rightarrow 0, \mathcal{D}_{D}(y)$ increases for intermediate values of $y$; as a result (D) boundary conditions induce an increase of the DoS at intermediate energies.

The low energy DoS presents a log-normal suppression :

$$
\overline{\varrho_{S}(\epsilon ; L)} \simeq \frac{16}{|\epsilon| \sqrt{2 \pi g L}} \mathrm{e}^{-\frac{2}{g L} \ln ^{2}(g /|\epsilon|)} \quad \text { for } \quad|\epsilon| \ll \epsilon_{c}=g \mathrm{e}^{-\sqrt{g L / 2}}
$$

and

$$
\overline{\varrho_{D}(\epsilon ; L)} \simeq \frac{4}{|\epsilon| \sqrt{2 \pi g L}} \mathrm{e}^{-\frac{1}{2 g L} \ln ^{2}(g /|\epsilon|)} \quad \text { for } \quad|\epsilon| \ll \epsilon_{c}^{\prime}=g \mathrm{e}^{-\sqrt{2 g L}}
$$

The DoS reaches its maximal value at $\epsilon_{*} \approx g \mathrm{e}^{-g L / 4}$ in the $(\mathrm{S})$ case and at $\epsilon_{*}^{\prime} \approx g \mathrm{e}^{-g L}$ in the (D) case.

The weaker log-normal suppression in the (D) case as compared to the $(\mathrm{S})$ case is due to the fact that for a given realisation of the disorder, the spectra for the two different kinds of 
boundary conditions are such that $\epsilon_{1}^{D}<\epsilon_{1}^{S}<\epsilon_{2}^{D}<\epsilon_{2}^{S}<\cdots$, where $\left\{\epsilon_{n}^{D, S}\right\}$ denotes the spectrum for boundary conditions of type (D) and (S) respectively.

Since $\mathcal{D}(y \rightarrow \infty) \simeq 1$, eqs. 1113 , we recover the Dyson singularity for intermediate energies, as expected :

$$
\overline{\varrho(\epsilon ; L)} \simeq L \varrho(\epsilon) \approx \frac{L g}{|\epsilon| \ln ^{3}(g /|\epsilon|)} \quad \text { for } \quad \epsilon_{c} \ll|\epsilon| \ll g .
$$

At higher energies $|\epsilon| \gg g$ one should recover the free DoS : $\overline{\varrho(\epsilon ; L)} \simeq L / \pi$.

The average DoS $\overline{\varrho(\epsilon ; L)}$ is represented for various values of $L$ on figure 2 , For the lowest energies $\epsilon \lesssim \epsilon_{*} \sim g \mathrm{e}^{-g L}$ the DoS is suppressed in both cases. In the intermediate range $\epsilon_{*} \lesssim \epsilon \lesssim$ $\epsilon_{c} \sim g \mathrm{e}^{-\sqrt{g L}}$, the effect of the boundaries is to reduce the DoS in the (S) case but surprisingly to increase the DoS in the (D) case.

As $L$ is decreased the Dyson singularity is rapidly converted to a strong depletion of the low energy DoS. For (S) boundary conditions this occurs for a surprisingly relatively large length $L_{*} \sim 10 / \mathrm{g}$. For the (D) case the memory of the Dyson singularity persists up to smaller lengths since an increase of the low energy DoS is apparent up to $L_{*}^{\prime} \sim 1 / g$ (figure 2).
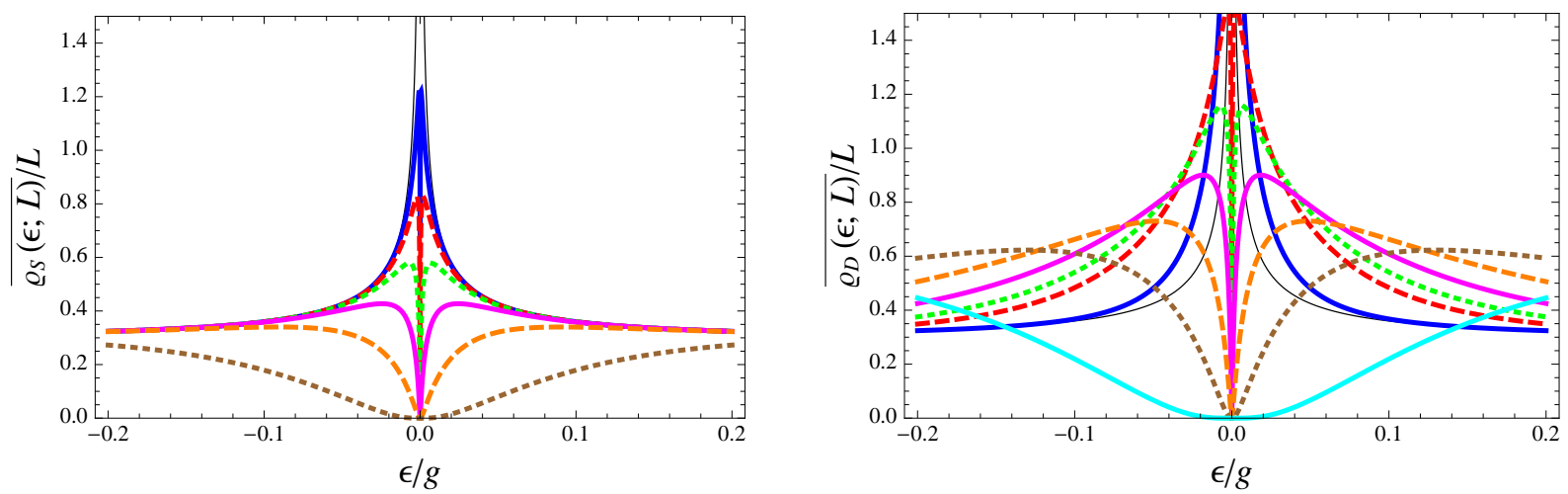

Figure 2: Average DoS per unit length for a finite $L$; parameter is $g=1$. Left : $(S)$ boundary conditions for sizes $L=\infty, 30,25,20,15,10$ \&5 5. Right: (D) boundary conditions for sizes $L=\infty, 12,6,5,4,3,2 \& 1$.

\section{Conclusion}

By using known results on the energy-levels distributions $\mathcal{W}_{n}(\epsilon ; L)$ we have obtained the average density of states of a random Dirac Hamiltonian on a bounded domain $[0, L]$. For $g L \gg 1$, we have seen that the DoS does not feel the boundary as energies become larger than the energy scale $\epsilon_{c} \sim g \mathrm{e}^{-\sqrt{g L}}$. In the intermediate range $\epsilon_{*} \lesssim \epsilon \lesssim \epsilon_{c}$, where $\epsilon_{*} \sim g \mathrm{e}^{-g L}$, the DoS may be diminished by boundaries, (S) case, or increased, (D) case. For the lowest energies the DoS presents a log-normal suppression for both kind of boundary conditions.

Note that the effect of one boundary condition was already studied in Ref. [10] where the average local DoS on a semi-infinite line was computed by the Berezinskuii technique. These authors found an increase of the local DoS by a factor $\ln (g /|\epsilon|) \gg 1$ close to the boundary (at a distance much smaller than the scale $\left.g^{-1} \ln ^{2}(g /|\epsilon|)\right)$. Here, by imposing boundary conditions at the two sides of finite interval, we have shown that boundary conditions can induce both an increase or a decrease of the DoS.

It is interesting to interpret our result within the classical diffusion problem. The $(\mathrm{S})$ boundary corresponds to diffusion in a random force field with two absorbing boundaries while the (D) case corresponds to one reflecting boundary and one absorbing. The return probability 
is the Laplace transform of the DoS, therefore the log-normal suppression of the average DoS coincides to a log-normal decay of the average return probability $\int_{0}^{L} \mathrm{~d} x \overline{P(x, t \rightarrow \infty \mid x, 0)} \sim$ $\exp -\frac{1}{g L} \ln ^{2}\left(g^{2} t\right)$.

To close this paper, let us examine the relation between our main result (915) with the localisation properties. It is only below the crossover energy $\epsilon_{c} \sim g \mathrm{e}^{-\sqrt{g L}}$ that eigenstates do feel the boundaries. The transition corresponds to the case where the localisation length is of order of the system size $\xi_{\epsilon_{c}} \sim L$, therefore this criterion suggests that the eigenstate of energy $\epsilon$ is localised on a scale $\xi_{\epsilon} \sim g^{-1} \ln ^{2}(g /|\epsilon|)$.

The question of localisation was studied in several works (Refs. [28, 29] for a tight binding Hamiltonian with random hoppings and in Ref. [17 for the continuous supersymmetric Hamiltonian) that have demonstrated the vanishing of the Lyapunov exponent ${ }^{4}$ at the band edge $\gamma(\epsilon \rightarrow 0) \sim g \ln ^{-1}(g /|\epsilon|)$. The Lyapunov exponent provides another possible definition of the inverse localisation length $\widetilde{\xi}_{\epsilon} \stackrel{\text { def }}{=} 1 / \gamma$, therefore much shorter than the previous one : $\widetilde{\xi}_{\epsilon} \sim g^{-1} \ln (g /|\epsilon|) \ll \xi_{\epsilon}$. In other terms the Lyapunov exponent analysis would put the "localisation threshold" 5 at $\epsilon_{*} \sim g \mathrm{e}^{-g L}$, much below than $\epsilon_{c} \sim g \mathrm{e}^{-\sqrt{g L}}$.

The existence of two characteristic length scales was pointed out in several works [17, 3, 5, 10, These authors have found that the average Green's function (i.e. the two point correlation function) decays over ${ }^{6} \xi_{\epsilon} \sim g^{-1} \ln ^{2}(g /|\epsilon|)$. The existence of two length scales was interpreted by Fisher [3] as a consequence of fluctuations (see also [5]). The "typical" localisation length $\widetilde{\xi}_{\epsilon}$ characterises the decay of a typical wave function (more precisely $\widetilde{\xi}_{\epsilon}$ is related to the average of the logarithm of the wave function) and the "average" localisation length $\xi_{\epsilon}$ controls the decay of the average correlation functions. Since we have considered an average quantity $\overline{\varrho(\epsilon ; L)}$, the fact that we have extracted the scale $\xi_{\epsilon}$ is consistent with Fisher's argument.

Several arguments support the existence of a delocalisation transition at $\epsilon \rightarrow 0$. Yet they are of quite different nature and it is interesting to provide a brief review. The first four arguments are bulk properties of the model. ( $i$ ) The Lyapunov exponent vanishes at low energies: $\gamma(\epsilon)=1 / \widetilde{\xi}_{\epsilon} \rightarrow 0$ for $\epsilon \rightarrow 0$ [17]. (ii) The calculation of the average Green's function [17, [5, 10]. (iii) The DC conductivity of the model was computed at the Dirac point in Ref. 1, 2] and was found to be finit 9 , (iv) The statistical properties of the zero mode wave function [33, 9, 34] indicate long range power law correlations. Another two arguments are obtained from scattering analysis : $(v)$ The distribution of the transmission probability through a finite slab of length $L$ at zero energy was obtained in Ref. [35] ; in particular the average transmission decreases like $1 / \sqrt{L}$, that is slower than the behaviour $1 / L$ for a quasi-1d weakly-disordered conducting wire. (vi) The time delay distribution presents a log-normal distribution at zero energy [35, 36] $P_{L}(\tau \rightarrow \infty) \sim \frac{1}{\tau \sqrt{L}} \exp -\frac{1}{8 g L} \ln ^{2}(g \tau)$, with moments diverging with the length of the disordered region 8 [36] $\overline{\tau(0)}=2 L$ and $\overline{\tau(0)^{n}} \sim g^{-n} \mathrm{e}^{2 n^{2} g L}$. Finally, two arguments consider the problem

\footnotetext{
${ }^{4}$ We recall that the Lyapunov exponent characterises the exponential growth rate of the envelope of the wave function [30, 13] $\gamma \stackrel{\text { def }}{=} \lim _{x \rightarrow \infty} \frac{\Xi(x)}{x}$ where $\Xi$ controls the envelope of the wave function $\psi(x)=\mathrm{e}^{\Xi(x)} \times$ oscillations.

${ }^{5}$ Note that in the scaling theory of localisation [31 the notion of a localisation threshold (or mobility edge) designates the energy separating localised and delocalised states for the infinite system. Within this usual terminology the model we are studying is always in a localised phase apart strictly at $\epsilon=0$. What we denote here by "localisation threshold" is the energy separating localised and delocalised states for a finite system size $L$. With this definition there can not be a sharp localisation transition, due to fluctuations over disorder realisations.

${ }^{6}$ Note that a related quantity first obtained Refs. [15, 16] is the propagator, the inverse Laplace transform of the Green's function, or more precisely the average conditional probability $\overline{P\left(x, t \mid x^{\prime}, 0\right)}=\overline{\phi_{0}(x) \phi_{0}\left(x^{\prime}\right)^{-1}\left\langle x\left|\mathrm{e}^{-t H_{+}}\right| x^{\prime}\right\rangle}$ of the related Fokker-Planck equation, where $\phi_{0}(x)$ is the zero mode of the supersymmetric Hamiltonian $H_{+}$; the result of these works was reproduced in Ref. [32] from stochastic Riccati analysis and in Ref. [19] with the real space renormalisation group.

7 In the localised phase in $1 \mathrm{~d}$, the AC conductivity vanishes at small frequency according to Mott's law $\operatorname{Re} \sigma(\omega) \sim \omega^{2} \ln ^{2} \omega[30$.

8 This suggests that a particle of energy $\epsilon \approx 0$ injected in a disordered region of size $L$ will spread over the full interval and remain trapped a very long time depending on $L$; whereas in a strongly localised phase the particle
} 
on a finite interval taking into account boundary conditions : (vii) The study of extreme value statistics of energy levels reveals spectral correlations for $\epsilon \rightarrow 0$ [25], whereas a localised phase is characterised by absence of level correlations [38. (viii) Finally the present work has shown the influence of the boundary on $\overline{\varrho(\epsilon \rightarrow 0 ; L)}$ due to delocalisation.

It remains an interesting issue to go beyond this analysis of the localisation characterised by the two lengths $\widetilde{\xi}_{\epsilon}$ and $\xi_{\epsilon}$, and comprehend better the role of fluctuations, for example for the DoS $\varrho(\epsilon ; L)$. Another challenging issue would be to extend our results to two dimensions. Since our method is specific to one-dimensional systems, such an extansion would necessitate the development of other methods. This might be of interest due to the recent revival of twodimensional random Dirac Hamiltonian physics, motivated by the study of Graphene.

\section{Acknowledgements}

It is our pleasure to acknowledge Alain Comtet for stimulating discussions, and in particular for pointing out the relation discussed in appendix C. Moreover, we thank Pierre Le Doussal for discussions.

\section{A Extreme value spectral statistics}

We give the first distributions introduced in the text. We compute the integral (6) using the residue theorem. This requires the expansion of the denominator near the pole $\kappa_{n}=-\frac{\pi^{2}}{4}(2 n+$ $1)^{2}, n \in \mathbb{N}: \cosh \sqrt{q}=\frac{(-1)^{n}}{\pi(2 n+1)}\left(q-\kappa_{n}\right)\left[1+\frac{q-\kappa_{n}}{-4 \kappa_{n}}+\frac{12+4 \kappa_{n}}{6}\left(\frac{q-\kappa_{n}}{-4 \kappa_{n}}\right)^{2}+\frac{10+4 \kappa_{n}}{2}\left(\frac{q-\kappa_{n}}{-4 \kappa_{n}}\right)^{3}+\cdots\right]$. Some algebra gives :

$$
\begin{aligned}
\pi_{1}(y) & =\sum_{n=0}^{\infty}(-1)^{n} \pi(2 n+1) \mathrm{e}^{-\frac{\pi^{2}}{4}(2 n+1)^{2} y} \\
& =\frac{1}{\sqrt{\pi} y^{3 / 2}} \sum_{n=0}^{\infty}(-1)^{n}(2 n+1) \mathrm{e}^{-(2 n+1)^{2} / 4 y} \\
\pi_{2}(y) & =\sum_{n=0}^{\infty}\left[\pi^{2}(2 n+1)^{2} y-2\right] \mathrm{e}^{-\frac{\pi^{2}}{4}(2 n+1)^{2} y} \\
& =\frac{4}{\sqrt{\pi} y^{3 / 2}} \sum_{n=0}^{\infty}(-1)^{n+1} n^{2} \mathrm{e}^{-n^{2} / y} \\
\pi_{3}(y) & =\sum_{n=0}^{\infty}(-1)^{n} \pi(2 n+1)\left[\frac{\pi^{2}(2 n+1)^{2}}{2} y^{2}-3 y+\frac{1}{2}\right] \mathrm{e}^{-\frac{\pi^{2}}{4}(2 n+1)^{2} y} \\
& =\frac{1}{2 \sqrt{\pi} y^{3 / 2}} \sum_{n=0}^{\infty}(-1)^{n+1}(2 n+1)\left[(2 n+1)^{2}-1\right] \mathrm{e}^{-(2 n+1)^{2} / 4 y} \\
\pi_{4}(y) & =\sum_{n=0}^{\infty}\left[\frac{\pi^{4}(2 n+1)^{4}}{6} y^{3}-2 \pi^{2}(2 n+1)^{2} y^{2}+2\left(\frac{\pi^{2}(2 n+1)^{2}}{3}+1\right) y-\frac{4}{3}\right] \mathrm{e}^{-\frac{\pi^{2}}{4}(2 n+1)^{2} y} \\
& =\frac{8}{3 \sqrt{\pi} y^{3 / 2}} \sum_{n=0}^{\infty}(-1)^{n} n^{2}\left(n^{2}-1\right) \mathrm{e}^{-n^{2} / y}
\end{aligned}
$$

would only explore a typical size $\widetilde{\xi}_{\epsilon}$ what is reflected in the fact that the time delay distribution reaches a limit distribution $\lim _{L \rightarrow \infty} P_{L}(\tau)=P_{\infty}(\tau)$ [37]. 
(the distribution $\pi_{1}(y)$ was already obtained in Ref. [7] where the energy $\epsilon_{1}$ is interpreted as the gap of a spin chain. It is also related to the distribution of the random bond lengths in the real space renormalisation group procedure [3, 6, 19, 27]; $\pi_{2}(y)$ and $\pi_{4}(y)$ are given in Ref. [25]).

Expressions (20|22 24 26) are obtained from eqs. (19)21 23 25) by using the Poisson formulae given in the next appendix and $\pi_{3}(y)=\left(-2 y^{2} \frac{\mathrm{d}}{\mathrm{d} y}-3 y+\frac{1}{2}\right) \pi_{1}(y), \pi_{2}(y)=-2\left(2 y \frac{\mathrm{d}}{\mathrm{d} y}+\right.$ 1) $\sum_{n=0}^{\infty} \mathrm{e}^{-\frac{\pi^{2}}{4}(2 n+1)^{2} y}$ and $\pi_{4}(y)=\left(\frac{8}{3} y^{3} \frac{\mathrm{d}^{2}}{\mathrm{~d} y^{2}}+8\left(y^{2}-\frac{y}{3}\right) \frac{\mathrm{d}}{\mathrm{d} y}+2 y-\frac{4}{3}\right) \sum_{n=0}^{\infty} \mathrm{e}^{-\frac{\pi^{2}}{4}(2 n+1)^{2} y}$.

\section{A.1 Generating function}

We propose now a method that allows for a systematic determination of the distributions $\pi_{n}(y)$. Let us introduce the generating function

$$
\mathcal{G}(z, y)=\sum_{n=1}^{\infty} z^{n} \pi_{n}(y)=z \int_{\mathcal{B}} \frac{\mathrm{d} q}{2 \mathrm{i} \pi} \frac{\mathrm{e}^{q y}}{\cosh \sqrt{q}-z}
$$

where summation was performed in the convergence radius $|z|<1$. The function arccos is single valued in the convergence disk, therefore we can write the poles of the integrand as $q_{n}=-(n \pi+\arccos z)^{2}$ with $n \in \mathbb{N}$. We compute the residues by using $\frac{1}{2 \sqrt{q_{n}}} \sinh \sqrt{q_{n}}=$ $(-1)^{n} \frac{\sqrt{1-z^{2}}}{2(n \pi+\arccos z)}$ (it is helpful to notice that $\arccos \left(x \pm \mathrm{i}^{+}\right)=\arccos (x) \mp \mathrm{i} 0^{+}$for $x$ real in $[-1,+1])$, whence

$$
\mathcal{G}(z, y)=\frac{z}{y} \frac{\partial}{\partial z} \sum_{n=0}^{\infty}(-1)^{n} \mathrm{e}^{-(n \pi+\arccos z)^{2} y}
$$

In order to apply the Poisson summation formula (32) and get the generating function of the distributions with odd indices, we exploit the symmetry with respect to a change in sign of the argument $\sum_{n=0}^{\infty}(-1)^{n} \mathrm{e}^{-(n \pi+\arccos z)^{2} y}=-\sum_{n=-\infty}^{-1}(-1)^{n} \mathrm{e}^{-(n \pi+\arccos (-z))^{2} y}$ :

$$
\begin{aligned}
\mathcal{O}(z, y) & =\frac{\mathcal{G}(z, y)-\mathcal{G}(-z, y)}{2}=\frac{z}{2 y} \frac{\partial}{\partial z} \sum_{n=-\infty}^{+\infty}(-1)^{n} \mathrm{e}^{-(n \pi+\arccos z)^{2} y} \\
& =\frac{z}{y} \frac{\partial}{\partial z} \frac{1}{\sqrt{\pi y}} \sum_{n=0}^{\infty} T_{2 n+1}(z) \mathrm{e}^{-\frac{(n+1 / 2)^{2}}{y}} .
\end{aligned}
$$

Here the $T_{n}(z)$ are the Chebychev polynomials of the first kind $T_{n}(x)=\cos (n \arccos (x))$. They may be rewritten as

$$
T_{n}(x)=\frac{n}{2} \sum_{k=0}^{\lfloor n / 2\rfloor}(-1)^{k} \frac{(n-k-1) !}{k !(n-2 k) !}(2 x)^{n-2 k}
$$

where $\lfloor x\rfloor$ is the integer part. Upon insertion into $(29)$ we obtain

$$
\frac{1}{z} \mathcal{O}(z, y)=\sum_{n=0}^{\infty} z^{2 n} \pi_{2 n+1}(y)=\frac{1}{\sqrt{\pi} y^{3 / 2}} \sum_{n=0}^{\infty}(-1)^{n}(2 n+1) \mathrm{e}^{-\frac{(n+1 / 2)^{2}}{y}} \sum_{k=0}^{n}(-1)^{k}\left(\begin{array}{c}
n+k \\
n-k
\end{array}\right)(2 z)^{2 k} .
$$

Using that $\left(\begin{array}{c}n \\ p\end{array}\right)=0$ for $p>n$, we may relax the constraint on the summation with respect to $k$ and extract (7a). Note that eq. (7a) allows to recover (20) and (24).

In order to evaluate $\pi_{n}(y)$ with $n=2 k$ recall that we deal with a distribution of $n$ positive i.i.d. random variables. It follows that we may obtain $\pi_{2 k}(y)$ by convolution of $\pi_{2 k-1}(y)$ and $\pi_{1}(y): \pi_{2 k}(y)=\int_{0}^{y} \mathrm{~d} x \pi_{2 k-1}(x) \pi_{1}(y-x)$. The integration is fairly cumbersome but one may verify that it yields $(7 \mathrm{~b})$. This completes the computation of $\pi_{n}(y)$ for all positive integers $n$ as announced in the main text. One may verify that the summation of all $\pi_{2 k}(y)$ (or $\pi_{2 k+1}(y)$ ) yields the densities given by eq. (15). 


\section{B Two useful Poisson formulae}

Let us start by recalling the well-known Poisson formula $\sum_{n \in \mathbb{Z}} f(n)=\sum_{n \in \mathbb{Z}} \hat{f}(2 \pi n)$ for any function $f(x)$ defined on $\mathbb{R}$, with $\hat{f}(k)=\int_{\mathbb{R}} \mathrm{d} x \mathrm{e}^{-\mathrm{i} k x} f(x)$ its Fourier transform. Applying this formula we obtain

$$
\sum_{n \in \mathbb{Z}} \mathrm{e}^{2 \mathrm{i} \pi n \eta} \mathrm{e}^{-\pi^{2}(n+\alpha)^{2} y}=\frac{1}{\sqrt{\pi y}} \sum_{n \in \mathbb{Z}} \mathrm{e}^{2 \mathrm{i} \pi(n-\eta) \alpha} \mathrm{e}^{-\frac{(n-\eta)^{2}}{y}},
$$

used for $\pi_{n}(y)$ with even indices (set $\eta=0$ and $\alpha=1 / 2$ ) and for the DoS (set $\eta=0$ or $\eta=1 / 2$ and $\alpha=0)$. For the distributions $\pi_{n}(y)$ with odd indices we need (with $\eta=\alpha=1 / 2$ )

$$
\sum_{n}(n+\alpha) \mathrm{e}^{2 \mathrm{i} \pi n \eta} \mathrm{e}^{-\pi^{2}(n+\alpha)^{2} y}=\frac{1}{\mathrm{i}(\pi y)^{3 / 2}} \sum_{n}(n-\eta) \mathrm{e}^{2 \mathrm{i} \pi(n-\eta) \alpha} \mathrm{e}^{-\frac{(n-\eta)^{2}}{y}} .
$$

\section{A probabilistic interpretation of the result for type (S) bound- ary conditions}

It is worth pointing that the function $\mathcal{D}_{S}(y)$ can be interpreted as the integrated distribution of the maximum of a Brownian excursion. Let us denote by $(x(t), 0 \leqslant t \leqslant 1)$ such an excursion. We establish a relation between the distribution of the maximum of such an excursion and the function $\mathcal{D}_{S}(y)$.

\section{C.1 Maximal height of a Brownian excursion}

Let us consider the distribution of the maximum $M$ of a Brownian excursion $(x(t), 0 \leqslant t \leqslant 1)$ (a Brownian bridge constraint to be positive). It can be written as a ratio of two path integrals :

$$
\text { Proba }[x(t) \leqslant M]=\lim _{x_{0} \rightarrow 0^{+}} \frac{\int_{x(0)=x_{0}}^{x(1)=x_{0}} \mathcal{D} x(t) \mathrm{e}^{-\frac{1}{2} \int_{0}^{1} \mathrm{~d} t \dot{x}^{2}} \prod_{t=0}^{1} \theta(x(t)) \theta(M-x(t))}{\int_{x(0)=x_{0}}^{x(1)=x_{0}} \mathcal{D} x(t) \mathrm{e}^{-\frac{1}{2} \int_{0}^{1} \mathrm{~d} t \dot{x}^{2}} \prod_{t=0}^{1} \theta(x(t))},
$$

where $x_{0}>0$ is a regulator. The ratio of path integrals may be rewritten as

$$
\operatorname{Proba}[x(t) \leqslant M]=\lim _{x_{0} \rightarrow 0^{+}} \frac{\left\langle x_{0}\left|\mathrm{e}^{-H_{1}}\right| x_{0}\right\rangle}{\left\langle x_{0}\left|\mathrm{e}^{-H_{0}}\right| x_{0}\right\rangle},
$$

where $H_{0}=-\frac{1}{2} \frac{\mathrm{d}^{2}}{\mathrm{~d} x^{2}}$ acts on functions defined on $\mathbb{R}^{+}$satisfying Dirichlet boundary condition at $x=0$ and $H_{1}=-\frac{1}{2} \frac{\mathrm{d}^{2}}{\mathrm{~d} x^{2}}$ acts on functions defined on $[0, M]$ with Dirichlet boundary conditions. It follows that [39, 40]

$$
\text { Proba }[x(t) \leqslant M]=\sqrt{\frac{2}{\pi}}\left(\frac{\pi}{M}\right)^{3} \sum_{n=1}^{\infty} n^{2} \mathrm{e}^{-\frac{1}{2}\left(\frac{n \pi}{M}\right)^{2}} .
$$

\section{C.2 First-exit time}

We consider a Brownian motion on $[0,1 / \sqrt{2}]$ with a reflecting boundary condition at $x=1 / \sqrt{2}$. We denote by $\tau$ the time at which $x(t)$ hits $x=0$ for the first time, starting from $x=1 / \sqrt{2}$, and $\tau_{x}$ the time needed to reach $x=0$, starting from $x . h(x, q)=\overline{\mathrm{e}^{-q \tau_{x}}}$ obeys the BFPE 
$\frac{1}{2} \frac{\mathrm{d}^{2}}{\mathrm{~d} x^{2}} h(x, q)=q h(x, q)$ with boundary conditions $h(0, q)=1$ and $\left.\partial_{x} h(x, q)\right|_{1 / \sqrt{2}}=0$ [41]. We easily find :

$$
h(x, q)=\frac{\cosh \sqrt{2 q}(x-1 / \sqrt{2})}{\cosh \sqrt{q}},
$$

therefore distribution of time $\tau$ is given by inverse Laplace transform of

$$
\overline{\mathrm{e}^{-q \tau}}=\frac{1}{\cosh \sqrt{q}} .
$$

We introduce the sum of $n$ i.i.d such variables : $y=\tau_{1}+\cdots+\tau_{n}$. The distribution of this variable was introduced in the text $\pi_{n}(y)=\int_{-\mathrm{i} \infty}^{+\mathrm{i} \infty} \frac{\mathrm{d} q}{2 \mathrm{i} \pi} \frac{\mathrm{e}^{q y}}{\cosh ^{n} \sqrt{q}}$, where we have shown that $\mathcal{D}_{S}(y)=$ $\sum_{n=1}^{\infty} \pi_{2 n}(y)=\frac{4}{\sqrt{\pi} y^{3 / 2}} \sum_{n=1}^{\infty} n^{2} \mathrm{e}^{-n^{2} / y}$. Comparing with 36 we seen that the sum of these distributions coincides with the cumulative distribution of the Brownian excursion 9

$$
\mathcal{D}_{S}(y)=\operatorname{Proba}[x(t) \leqslant M=\pi \sqrt{y / 2}] .
$$

It would be interesting to know whether this remark is purely accidental or not.

\section{References}

[1] A. A. Gogolin and V. I. Mel'nikov, Conductivity of one-dimensional metal with half-filled band, Sov. Phys. JETP 46, 369 (1977).

[2] A. A. Gogolin, Electron localization and hopping conductivity in one-dimensional disordered systems, Phys. Rep. 86(1), 1-53 (1982).

[3] D. S. Fisher, Random antiferromagnetic quantum spin chains, Phys. Rev. B 50(6), 3799 (1994).

[4] R. H. McKenzie, Exact Results for Quantum Phase Transitions in Random XY Spin Chains, Phys. Rev. Lett. 77(23), 4804 (1996).

[5] L. Balents and M. P. A. Fisher, Delocalization transition via supersymmetry in one dimension, Phys. Rev. B 56(20), 12970 (1997).

[6] D. S. Fisher, Critical behavior of random transverse-field Ising spin chains, Phys. Rev. B 51(10), 6411-6461 (1995).

[7] D. S. Fisher and A. P. Young, Distributions of gaps and end-to-end correlations in random transverse-field Ising spin chains, Phys. Rev. B 58(14), 9131-9141 (Oct 1998).

[8] D. S. Fisher, P. Le Doussal, and C. Monthus, Random walks, reaction-diffusion and nonequilibrium dynamics of spin chains in one-dimensional random environments, Phys. Rev. Lett. 80(16), 3539 (1998).

[9] D. G. Shelton and A. M. Tsvelik, Effective theory for midgap states in doped spin-ladder and spin Peierls systems: Liouville quantum mechanics, Phys. Rev. B 57(22), 14242 (1998).

[10] M. Steiner, M. Fabrizio, and A. O. Gogolin, Random mass Dirac fermions in doped spinPeierls and spin-ladder systems: one-particle properties and boundary effects, Phys. Rev. B 57(14), 8290 (1998).

\footnotetext{
${ }^{9}$ The fact that this sum can be interpreted as a cumulative distribution is related to the fact that the average density of the variables $\tau_{n}$ 's is unity.
} 
[11] H. Takayama, Y. R. Lin-Liu, and K. Maki, Continuum model for solitons in polyacetylene, Phys. Rev. B 21(6), 2388 (1980).

[12] A. A. Ovchinnikov and N. S. Erikmann, Density of states in a one-dimensional random potential, Sov. Phys. JETP 46, 340 (1977).

[13] I. M. Lifshits, S. A. Gredeskul, and L. A. Pastur, Introduction to the theory of disordered systems, John Wiley \& Sons, 1988.

[14] Ya. G. Sinai, The limit behavior of random walks in a one-dimensional random environment, Theory of Prob. and Appl. 27(2), 247 (1982).

[15] A. O. Golosov, Commun. Math. Phys. 92, 491 (1984), Also Sov. Math. Dokl. 28 (1983) 18.

[16] H. Kesten, The limit distribution of Sinai's random walk in random environment, Physica 138A, 299 (1986).

[17] J.-P. Bouchaud, A. Comtet, A. Georges, and P. Le Doussal, Classical diffusion of a particle in a one-dimensional random force field, Ann. Phys. (N.Y.) 201, 285-341 (1990).

[18] C. Aslangul, N. Pottier, and D. Saint-James, Random walk in a one-dimensional random medium, Physica 164A, 52 (1990).

[19] P. Le Doussal, C. Monthus, and D. S. Fisher, Random walkers in one-dimensional random environments: Exact renormalization group analysis, Phys. Rev. E 59(5), 4795 (1999).

[20] C. Monthus and P. Le Doussal, Localization of thermal packets and metastable states in the Sinai model, Phys. Rev. E 65(6), 066129 (2002).

[21] M. Bocquet, Some spectral properties of the one-dimensional disordered Dirac equation, Nucl. Phys. B [FS] 546, 621 (1999).

[22] A. Comtet, J. Desbois, and C. Monthus, Localization properties in one-dimensional disordered supersymmetric quantum mechanics, Ann. Phys. (N.Y.) 239, 312-350 (1995).

[23] A. Chodos, R. L. Jaffe, K. Johnson, C. B. Thorn, and V. F. Weisskopf, New extended model of hadrons, Phys. Rev. D 9(12), 3471-3495 (1974).

[24] M. Antoine, A. Comtet, and M. Knecht, Heat Kernel expansion for fermionic billiards in an external magnetic field, J. Phys. A: Math. Gen. 23, L35 (1990).

[25] C. Texier, Individual energy level distributions for one-dimensional diagonal and offdiagonal disorder, J. Phys. A: Math. Gen. 33, 6095-6128 (2000).

[26] C. Texier and C. Hagendorf, One-dimensional classical diffusion in a random force field with weakly concentration absorbers, Europhys. Lett. 86, 37011 (2009).

[27] P. Le Doussal, Sinai model in presence of dilute absorbers, preprint, condmat/arXiv:0906.0267 (2009).

[28] T. P. Eggarter and R. Riedinger, Singular behavior of tight-binding chains with off-diagonal disorder, Phys. Rev. B 18(2), 569 (1978).

[29] T. Ziman, Localization and spectral singularities in random chains, Phys. Rev. Lett. 49(5), 337 (1982). 
[30] T. N. Antsygina, L. A. Pastur, and V. A. Slyusarev, Localization of states and kinetic properties of one-dimensional disordered systems, Sov. J. Low Temp. Phys. 7(1), 1-21 (1981).

[31] B. Kramer and A. MacKinnon, Localization: theory and experiment, Rep. Prog. Phys. 56, 1469 (1993).

[32] A. Comtet and D. S. Dean, Exact results on Sinai's diffusion, J. Phys. A: Math. Gen. 31, 8595 (1998).

[33] K. Broderix and R. Kree, Thermal equilibrium with the Wiener potential: testing the replica variational approximation, Europhys. Lett. 32, 343-348 (1995).

[34] A. Comtet and C. Texier, One-dimensional disordered supersymmetric quantum mechanics: a brief survey, in Supersymmetry and Integrable Models, edited by H. Aratyn, T. D. Imbo, W.-Y. Keung, and U. Sukhatme, Lecture Notes in Physics, Vol. 502, pages 313-328, Springer, 1998, Proceedings of a workshop held at Chicago, IL, USA, 12-14 June 1997 (also available as cond-mat/97 07313$)$.

[35] M. Steiner, Y. Chen, M. Fabrizio, and A. O. Gogolin, Statistical properties of localizationdelocalization transition in one dimension, Phys. Rev. B 59(23), 14848 (1999).

[36] C. Texier, Quelques aspects du transport quantique dans les systèmes désordonnés de basse dimension, $\mathrm{PhD}$ thesis, Université Paris 6, 1999, available at http://www.lptms.upsud.fr/membres/texier/research.html.

[37] C. Texier and A. Comtet, Universality of the Wigner time delay distribution for onedimensional random potentials, Phys. Rev. Lett. 82(21), 4220-4223 (1999).

[38] S. A. Molčanov, The Local Structure of the Spectrum of the One-Dimensional Schrödinger Operator, Commun. Math. Phys. 78, 429 (1981).

[39] K. L. Chung, Excursions in Brownian motion, Arkiv för Matematik 14(1/2), 155 (1976).

[40] P. Biane, J. Pitman, and M. Yor, Probability laws related to the Jacobi $\theta$ and Riemann $\zeta$ functions and Brownian excursions, Bull. Amer. Math. Soc. (N.S.) 38(4), 435 (2001).

[41] C. W. Gardiner, Handbook of stochastic methods for physics, chemistry and the natural sciences, Springer, 1989. 